\title{
Measuring Grain Boundary Character Distributions from 3D EBSD Data
}

\author{
J. Li and G.S. Rohrer
}

Materials Research Science and Engineering Center and Department of Materials Science and Engineering, Carnegie Mellon University, 5000 Forbes Avenue, Pittsburgh, PA 15213

The grain boundary character distribution (GBCD) is defined as the relative areas of grain boundaries distinguished by lattice misorientation and grain boundary plane normal, in units of multiples of a random distribution (MRD) [1]. Recently, the dual-beam focused ion beam (FIB) scanning electron microscope (SEM) has been used to automate the collection of serial sections of electron backscatter diffraction maps and the GBCD has been determined from these maps [2-4]. However, the method used in the earlier studies extracted information exclusively from the neighborhood of the triple junctions. As a result, it is possible that the sampling of grain boundary types is biased and that the resulting distribution differs from the true distribution. The main point of the current presentation is to present a more complete method for measuring the GBCD and compare the new results to those derived using the original method.

The GBCD calculation used for the prior work and the one described here are based on grain boundary line segments derived from parallel orientation maps. In the prior work, triple junctions (points where three line segments meet) on one layer were compared to triple junctions on adjacent layers [2-5]. When matching triplets were identified, they were connected by a triple line and the orientations of the grain boundary planes were then determined from the cross products of the triple line with each line segment. Grain boundary line segments not connected to triple lines are therefore ignored in the calculation of the GBCD. To overcome this problem, a new algorithm was developed that matches line segments. The algorithm considers the locations of the midpoint of segments on adjacent layers (labeled $\mathrm{M}_{1}$ and $\mathrm{M}_{2}$ in Fig. 1), as well as the misorientations across the segments. Segments that share lateral proximity and a similar misorientation are assumed to lie in the same grain boundary. In this case, the area between the two segments is tiled with three triangles (labeled 1, 2, and 3 in Fig. 1) and the orientation of each triangle and its area is added to the GBCD.

The efficacy of this procedure is illustrated in Fig. 2. In both parts of the figure, the line segments are color coded to show whether or not they were matched with segments in parallel layers. In Fig. $2 \mathrm{a}$, when the triple junction matching procedure is used, only $56 \%$ of the segments are matched. In Fig. $2 \mathrm{~b}$, where the new algorithm was used, $81 \%$ of the segments were matched. The majority of the newly added segments are associated with Sigma 3 grain boundaries and this alters the GBCD in a significant way. When the two methods are compared on a microstructure without extensive twinning, both methods produce comparable results.

References

[1] G.S. Rohrer, D.M. Saylor, B.S. El-Dasher, B.L. Adams, A.D. Rollett, and P. Wynblatt, Z. Metall., 95 (2004) 214.

[2] S.J. Dillon and G.S. Rohrer, J. Am. Ceram. Soc., 92 (2009) 1580.

[3] J. Li, S.J. Dillon and G.S. Rohrer, Acta Materialia, 57 (2009) 4304.

[4] G.S. Rohrer, J. Li, S. Lee, A.D. Rollett, M. Groeber, M.D. Uchic, Mat. Sci. Tech., 26 (2010) 661.

[5] Support from the MRSEC program of the NSF under Award DMR-0520425 is acknowledged. 

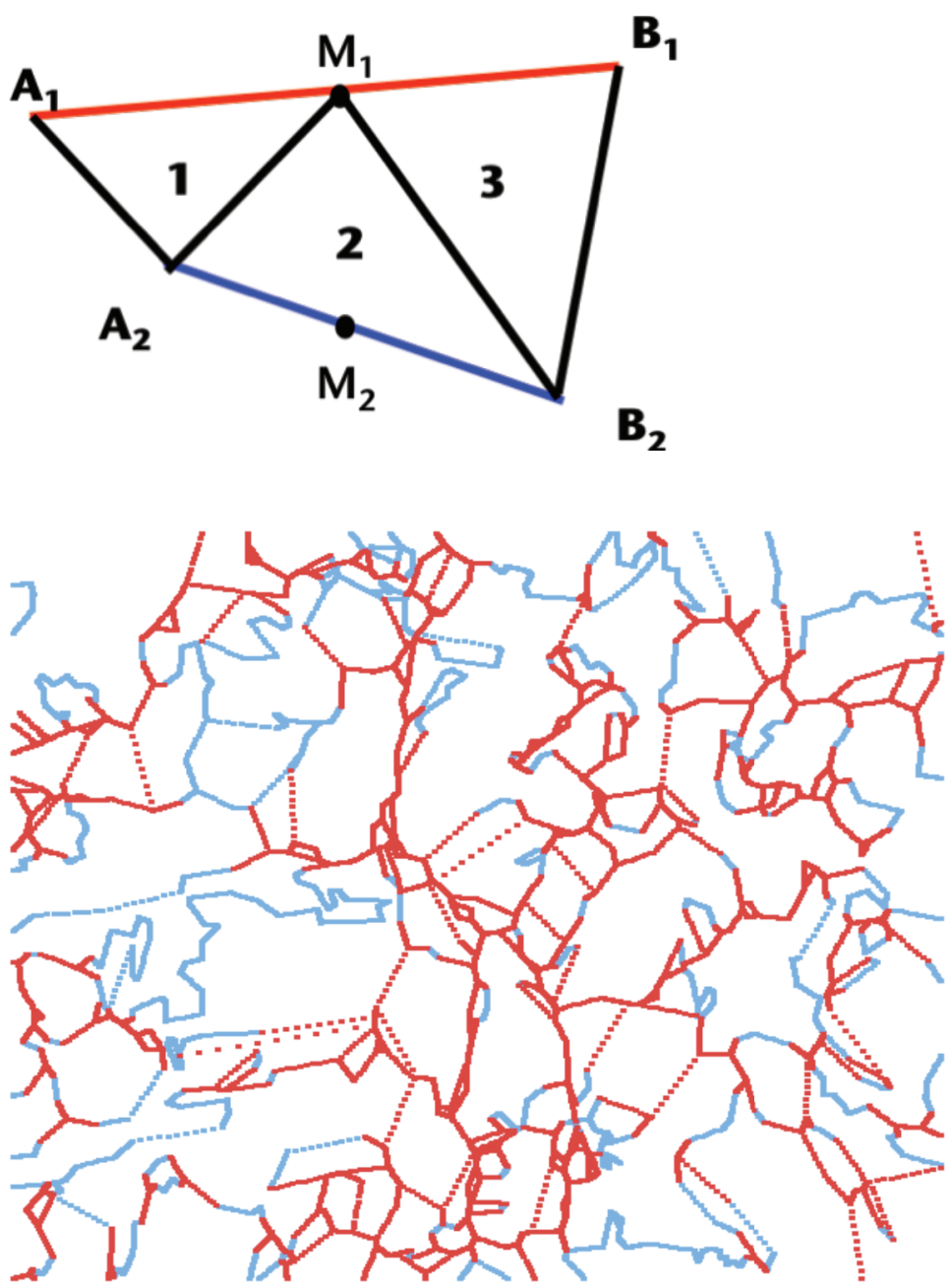

(a)

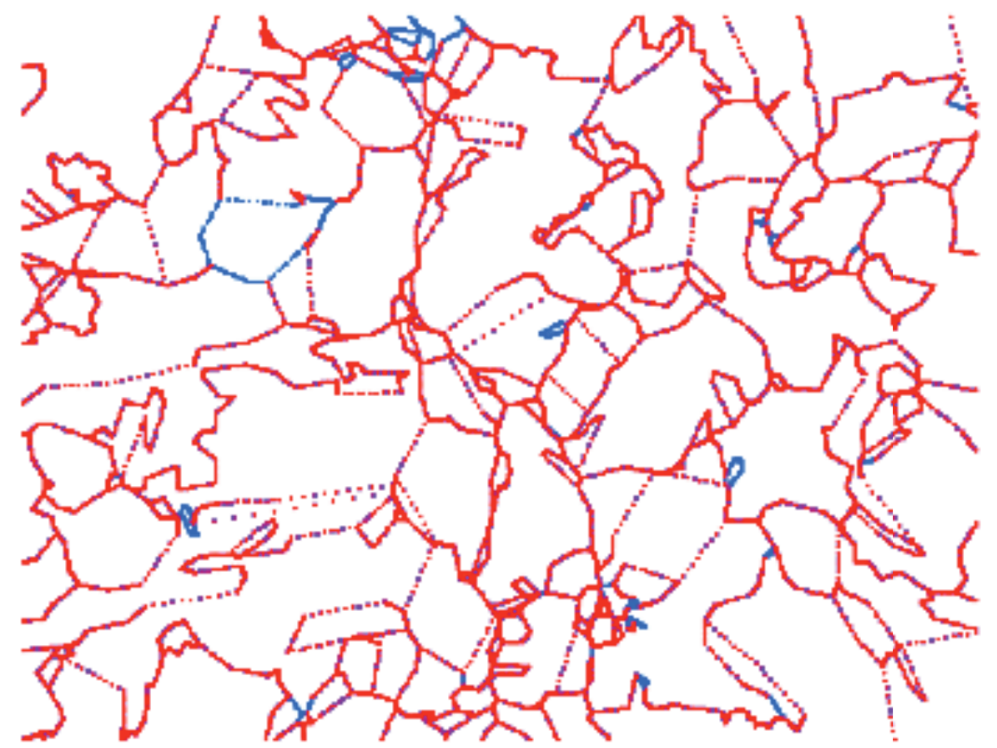

(b)
Figure 1. Schematic illustration of how line segments on adjacent layers are used to find grain boundary planes. Lines connect the end points $\left(A_{1}\right.$ to $A_{2}$ and $B_{1}$ to $\left.B_{2}\right)$ and then three triangles are formed by connecting a midpoint $\left(\mathrm{M}_{1}\right)$ to the endpoints on the adjacent layer $\left(\mathrm{A}_{2}\right.$ and $\left.\mathrm{B}_{2}\right)$.

Figure 2. Grain boundary line segment maps, derived form EBSD maps, from the same layer of high purity Ni. Each map has a horizontal dimension of 90 microns. (a) All line segments matched on an adjacent layer are colored red and those not included in the GBCD calculation are colored blue. This map was determined using the triple junction matching method used in previous reports [2-4]. (b) This map contains the same data and the grain boundary segments are colored in the same way. However, in this case the new segment matching algorithm was used and many more of the segments can be associated with segments on other layers belonging to the same boundary. 